\title{
La psicología del consumidor en Colombia: una revisión bibliométrica de las publicaciones científicas indexadas*
}

\section{Consumer psychology in Colombia: A bibliometric review of the indexed scholarly publications}

\author{
María Camila Maldonado Pinzón ${ }^{1} \underline{\mathrm{ORCID}}$, Andrés M. Pérez-Acosta ${ }^{2} \underline{\mathrm{ORCID}}$ \\ Universidad del Rosario \\ Bogotá, Colombia
}

Fecha correspondencia:

Recibido: septiembre 19 de 2018.

Aceptado: diciembre 9 de 2019.

Forma de citar:

Maldonado Pinzón, M. C., \& Pérez-

Acosta, A.M. (2020). La psicología

del consumidor en Colombia:

una revisión bibliométrica de

las publicaciones científicas

indexadas. Rev. CES Psico, 13(1),

153-165.

\section{Open access}

(c) Copyright

Licencia creative commons

Ética de publicaciones

Revisión por pares

Gestión por Open Journal System DOl: http://dx.doi.org/10.21615/ cesp.13.1.10

ISSN: 2011-3080

Sobre los autores:

* El presente trabajo se basó en el trabajo de grado como Psicóloga de la primera autora, bajo la dirección del segundo autor, en la Universidad del Rosario.

Comparte

7日G.

\section{Resumen}

La psicología del consumidor es un campo aplicado que se vale de las aproximaciones teóricas de la ciencia psicológica para entender el comportamiento del consumidor. Históricamente, la psicología del consumidor se ha desarrollado fundamentalmente en Estados Unidos y, en la actualidad, se ha fortalecido en otros países. Sin embargo, aún no se ha estudiado la contribución de Colombia al campo de la psicología del consumidor. Por tal motivo, se efectuó un análisis bibliométrico de la producción científica en psicología del consumidor publicada en español por investigadores afiliados a instituciones colombianas. Se seleccionó una muestra de 74 artículos académicos que abordan algún aspecto del área, publicados en revistas científicas indexadas en las siguientes bases de datos: Redalyc, DOAJ, Psicodoc, Scopus, Scielo, Web of Science y Google Scholar. Se utilizaron los siguientes descriptores de búsqueda: psicología, consumidor, Colombia, psicología del consumidor, comportamiento del consumidor y consumo. Se evidenció que la producción nacional ha crecido sostenidamente en las últimas décadas y que ya comienza a tener impacto por número de citaciones; no obstante, aún requiere de mayor colaboración internacional para su consolidación.

Palabras clave: Comportamiento del Consumidor, Psicología del Consumidor, Bibliometría, Revistas Científicas, Colombia.

\section{Abstract}

Consumer psychology is an applied field that uses theoretical approaches to psychological science to understand consumer behavior. Historically, consumer psychology has been developed primarily in the United States and now strengthened in other countries. However, the contribution of Colombia to the field has not been studied yet. For this reason, a bibliometric analysis of the academic production in consumer psychology was carried out in Spanish by authors affiliated to Colombian institutions. It was selected a sample of 74 academic articles that addressed certain aspect of the field, published in scientific journals indexed in the following databases: Redalyc, DOAJ, Psicodoc, Scopus, Scielo, Web of Science and Google Scholar. The following descriptors were used in the search: psychology, consumer, Colombia, consumer psychology, consumer behavior and consumption. It 


\section{Sobre los autores:}

1. Psicóloga egresada de la Universidad del Rosario (Bogotá, Colombia), con énfasis en psicología organizacional.

2. PhD. En Psicología. Psicólogo. Profesor Titular del Programa de Psicología, Escuela de Medicina y Ciencias de la Salud, Universidad del Rosario, Bogotá, Colombia. was showed that production has grown steadily in recent decades and this starts to impact by means of citations. However, this is a national production that still requires greater international collaboration for its consolidation.

Keywords: Consumer Behavior, Consumer Psychology, Bibliometrics, Scientific Journals, Colombia.

\section{Introducción}

La psicología del consumidor es un área en la que se aplican teorías y modelos de la psicología básica para la descripción, explicación y predicción de la conducta humana en el contexto de mercados reales (Sandoval, 1994). Según la División 23 de la American Psychological Association (2019), la psicología del consumidor es un campo aplicado que se vale de las aproximaciones teóricas de la ciencia psicológica para entender el comportamiento del consumidor.

Según Parrado (2013), la psicología del consumidor tiene sus raíces en la psicología industrial, a la que se reconocen sus inicios en diciembre de 1901, cuando Walter Dill Scott ofrece una conferencia en la que analiza las posibilidades de aplicación de la psicología al campo de la publicidad. Este evento se deriva de la Revolución Industrial previa que se propagó de Europa hacia Estados Unidos en el siglo XIX, fundamentalmente en las áreas del transporte, manufactura de metal, textil y agricultura, con profundos impactos en la estructura de la sociedad; lo que significó un incremento en los suministros alimenticios, materias primas y tecnologías más eficientes, que aumentaron el nivel de consumo de muchos productos. En consecuencia, se incrementó el interés por comprender e investigar cómo esta nueva producción afectaba los patrones de consumo de las personas (Jansson-Boyd, 2010).

Distintos enfoques clásicos de la psicología han estudiado la psicología del consumidor bajo sus supuestos, pero especialmente el conductismo ha generado investigaciones científicas de mercados que han aportado al conocimiento científico de este campo. John B. Watson desarrolló el primer plan de investigación científica aplicado al área de la psicología del consumidor (Parrado, 2013). Watson, durante su carrera académica basada en Pavlov, usó la técnica del condicionamiento clásico y estudió particularmente como persuadir a la gente de consumir otro tipo de productos distintos a los que se consumían en la época. Él creía que todos los individuos podían ser condicionados desde un principio por cualquier estímulo, y así mismo podían ser persuadidos para consumir un tipo específico de marca o producto (Jansson-Boyd, 2010).

Desde entonces, en el campo de la psicología del consumidor se han generado teorías, investigaciones empíricas y aplicaciones de los procesos psicológicos básicos a la publicidad y el mercadeo. Se ha contribuido a la creación de modelos teóricos que explican los procesos de decisión de las personas, los cuales se han alimentado de las teorías básicas de los procesos cognitivos humanos involucrados en las conductas de compra y consumo, con el fin de diseñar ambientes de servicio, estrategias de comunicaciones y construcción de marcas mejor posicionadas entre los consumidores (Sandoval-Escobar, 2018). En cuanto al aspecto metodológico, los psicólogos enfocados en el área del consumidor han utilizado distintas estrategias de investigación, tanto cuantitativas como cualitativas (Jansson-Boyd, 2010).

Todo estos ejercicios de investigación se han visto reflejados en la creación de revistas especializadas en el área como: Journal of Marketing Research (fundada en 1964), Journal of Consumer Research (fundada en 1974), Journal of Consumer Psychology (fundada en 
Pág 155

Aunque la psicología del consumidor se ha desarrollado fundamentalmente en Estados Unidos, otros países poco a poco se han ido abriendo paso en este campo. Específicamente, los países hispanohablantes (España y los países de Hispanoamérica) han originado en las últimas décadas un creciente número de publicaciones (Chilito et al., 2010).
1992), donde se han publicado estudios relativos a los procesos psicológicos involucrados en estrategias de publicidad y mercadeo, tales como percepción, memoria, aprendizaje, motivación, emoción, toma de decisiones, aspectos psicolingüísticos y psicosociales, como la influencia social y la persuasión (Chilito, Rodríguez, Plata, \& Pérez, 2010).

Aunque la psicología del consumidor se ha desarrollado fundamentalmente en Estados Unidos, otros países poco a poco se han ido abriendo paso en este campo. Específicamente, los países hispanohablantes (España y los países de Hispanoamérica) han originado en las últimas décadas un creciente número de publicaciones (Chilito et al., 2010).

Es importante mencionar que la psicología del consumidor es una disciplina que hace parte del campo interdisciplinario denominado "comportamiento del consumidor", al cual también aportan otras disciplinas como la economía, la antropología y las neurociencias, entre otras. Mientras la psicología busca una comprensión del proceso a través del cual los consumidores toman sus decisiones, las motivaciones y factores que las determinan, rechazando la universalidad de las mismas; el interés de la economía se centra en explicar el comportamiento colectivo, no el individual, y en el resultado de las decisiones más no en el proceso como tal (Raiteri, 2016). No obstante, estas diferencias entre los supuestos de cada disciplina, existe una relación de cooperación entre ambas, expresada en lo que Raiteri (2016) refiere como la psicología económica, que estudia los mecanismos psicológicos que hay detrás de determinados comportamientos económicos, se interesa en las preferencias, elecciones y decisiones y sus consecuencias respecto a la satisfacción de necesidades.

Hawkins (citado en Rodríguez, 2012) define el comportamiento del consumidor como el estudio de personas, grupos u organizaciones y los procesos que siguen para seleccionar, conseguir, usar y disponer de productos, servicios, experiencias o ideas que satisfacen sus necesidades, y los impactos que estos procesos tienen en el consumidor y la sociedad. De manera concreta, el comportamiento del consumidor se enfoca en la manera en que los consumidores y las familias o los hogares toman decisiones para gastar sus recursos disponibles (tiempo, dinero, esfuerzo) en artículos relacionados con el consumo. Eso incluye lo que compran, por qué lo compran, cuando, donde, con qué frecuencia lo compran y lo utilizan, cómo lo evalúan después de la compra, el efecto de estas evaluaciones sobre compras futuras y como lo desechan (Schiffman \& Lazar, 2010). En la medida en que este campo interdisciplinario también se enfoca en las acciones de cada individuo en el consumo, entonces la psicología del consumidor se inserta dentro de su espectro.

En el contexto regional hispanoamericano, Chilito et al. (2010) realizaron un análisis bibliométrico de la producción científica en psicología del consumidor publicada en lengua castellana y encontraron un aporte importante de autores colombianos (por ejemplo: Jim Walters, María Mercedes Botero, Marithza Sandoval, entre otros) y de revistas colombianas (como Suma Psicológica, Psicología desde el Caribe y Universitas Psychologica); sin embargo, aún no se ha estudiado de forma específica el aporte de Colombia a este campo del conocimiento.

Continuando con la misma estrategia metodológica, las bases de datos académicas podrían dar cuenta de las prácticas investigativas que han contribuido a fortalecer académicamente la actuación profesional de los psicólogos del consumidor en Colombia, tanto a título individual como en sus respectivos grupos de investigación e instituciones de afiliación. 


\section{Método}

La presente investigación se planteó como propósito general efectuar un análisis bibliométrico de la producción científica en psicología del consumidor publicada por investigadores afiliados a instituciones colombianas. En ese sentido, este estudio se define metodológicamente como descriptivo y de carácter retrospectivo.

\section{Muestra}

En primer lugar, se utilizó una técnica exploratoria de muestreo por conveniencia (muestreo en cadena o muestreo de bola de nieve) para la recolección de información piloto, bajo el supuesto de que no toda la información relevante para este estudio está necesariamente indexada en bases de datos. Después se realizó la búsqueda formal, utilizando los descriptores: psicología AND consumidor AND comportamiento AND consumo AND Colombia (en español y sin límite de tiempo) en las siguientes bases de datos: Redalyc (1102 resultados), Scielo (0 resultados), DOAJ (4 resultados), Psicodoc (23 resultados), Scopus (1 resultado), Web of Science (0 resultados) y Google Scholar (34200 resultados).

La muestra finalmente seleccionada de 74 artículos, que se pueden consultar en un enlace abierto en Google Drive ${ }^{1}$, incluye artículos que abordan el tema de psicología del consumidor publicados en revistas científicas, todas disponibles en bases de datos, es decir, indexadas y, además, con datos de impacto por citaciones.

Se excluyeron artículos que no fueran claramente derivados de investigación, que sus autores de correspondencia no tuvieran afiliación institucional colombiana y cuyo contenido no estuviera relacionado con psicología del consumidor. Adicionalmente, cuando en el artículo no se especificaba el autor de correspondencia o eran varios autores de correspondencia, éste se identificó con base en los siguientes criterios: estatus académico y orden de autoría.

\section{Procedimiento}

De cada uno de los 74 artículos seleccionados para el análisis bibliométrico, se extrajo información respecto a las siguientes variables: autores, autores de correspondencia (estos últimos analizados por aparte, pues son aquellos responsables directos de las investigaciones publicadas), año, institución de autor de correspondencia, ciudad, revista, tipo de artículo (investigación, revisión de literatura o reflexión teórica), tipo de colaboración (local, nacional, internacional y único autor).

A continuación, se realizó un análisis de la visibilidad e impacto por citaciones de los artículos en tres bases de datos académicas reconocidas internacionalmente: Google Scholar, Scopus y Web of Science.

Finalmente, se realizó un análisis de los ocho autores de correspondencia que han publicado con mayor frecuencia con relación a los datos sobre grupos de investigación registrados en Colciencias (GRUPLAC, vigentes en el año 2017) ${ }^{2}$ con el fin de conocer los grupos de investigación a los que están asociados, la clasificación de los grupos y la categoría individual como investigador de cada autor. 


\section{Resultados}

\section{Variables bibliográficas}

Como se observa en la Figura 1, los autores colombianos, de los artículos que conforman la muestra, más productivos en función de la frecuencia de publicación fueron Andrés M. Pérez-Acosta de la Universidad del Rosario (Bogotá), Marithza Sandoval Escobar de la Fundación Universitaria Konrad Lorenz (Bogotá), Idaly Barreto de la Fundación Universitaria Konrad Lorenz (Bogotá), Ana María Arboleda Arango de la Universidad Icesi (Cali), Andrea Velandia-Morales de la Fundación Universitaria Konrad Lorenz (Bogotá), Julio Eduardo Cruz de la Universidad de los Andes (Bogotá), Jim Walters de la Fundación Universitaria Konrad Lorenz (Bogotá) y María Mercedes Botero de la Universidad del Norte (Barranquilla).

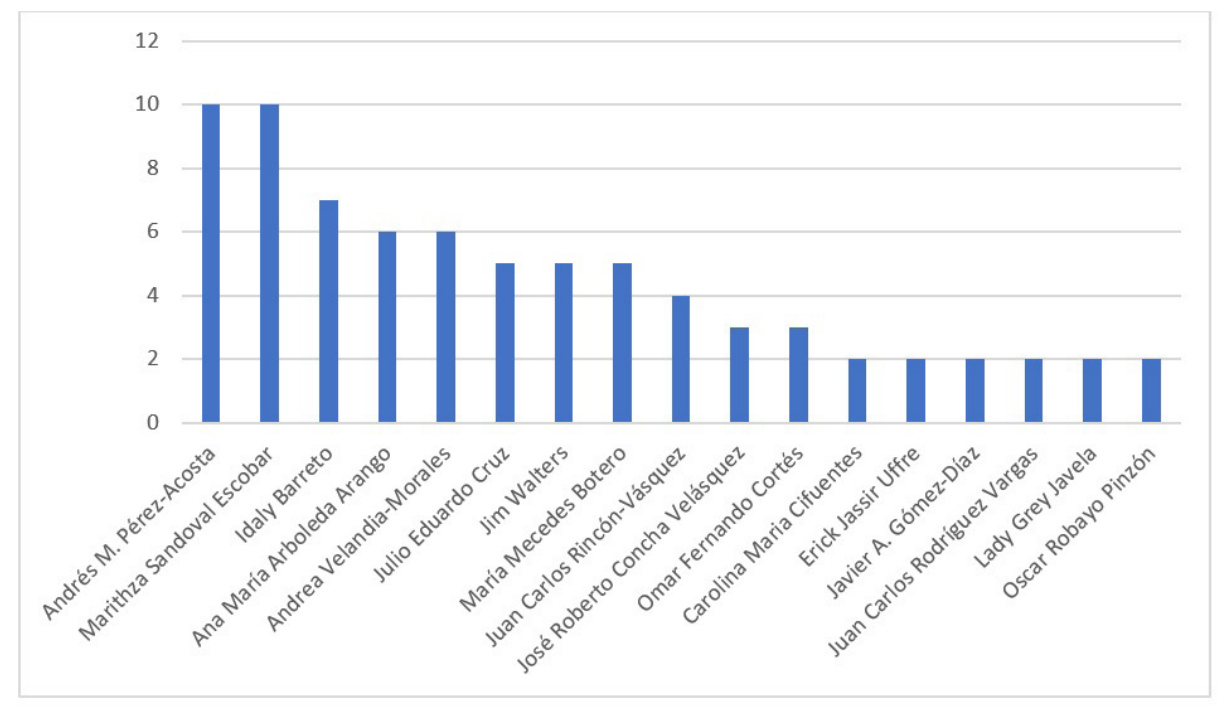

Figura 1. Autores según Frecuencias de Publicación

Como se muestra en la Figura 2, los autores de correspondencia que publicaron con más frecuencia fueron Marithza Sandoval Escobar con ocho publicaciones, Idaly Barreto con siete, Andrés M. Pérez-Acosta y Ana María Arboleda Arango con seis, María Mercedes Botero con cinco, Andrea Velandia-Morales con cuatro, Julio Eduardo Cruz con tres y, finalmente, Jim Walters con dos publicaciones.

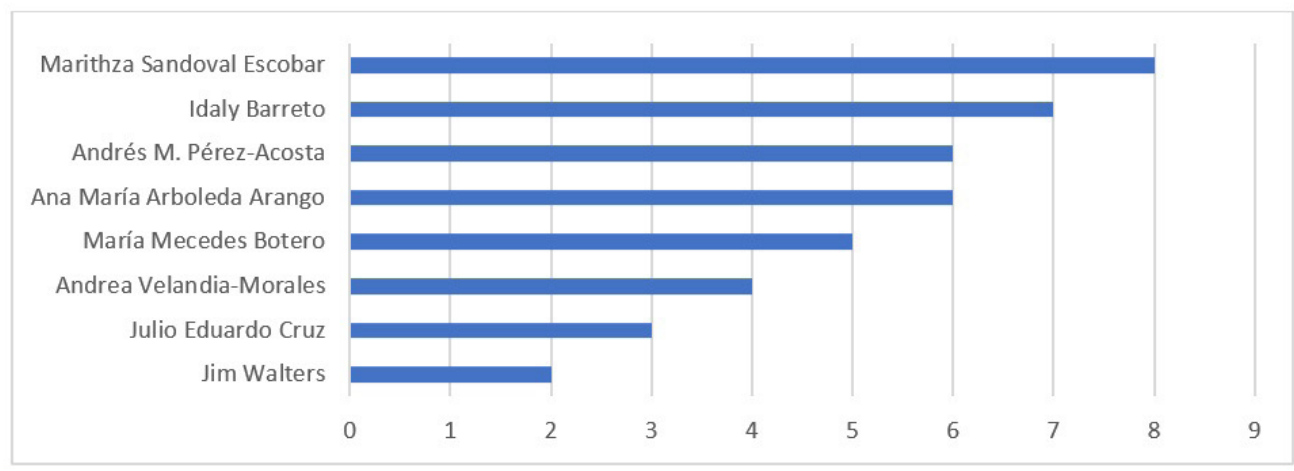

Figura 2. Autores de correspondencia según Frecuencias de Publicación

Respecto a los años de publicación, como se observa en la Figura 3, en 1978 se publicó el primer artículo de autores colombianos en la historia de la psicología del 
consumidor, en 1994 la frecuencia asciende a seis publicaciones, y en 2011 se evidencia la producción más alta con nueve artículos. En 2012 se observa una caída de productividad científica ya que solo se publicaron dos artículos, y finalmente el último año de producción científica publicada fue 2016 con una frecuencia de siete artículos.

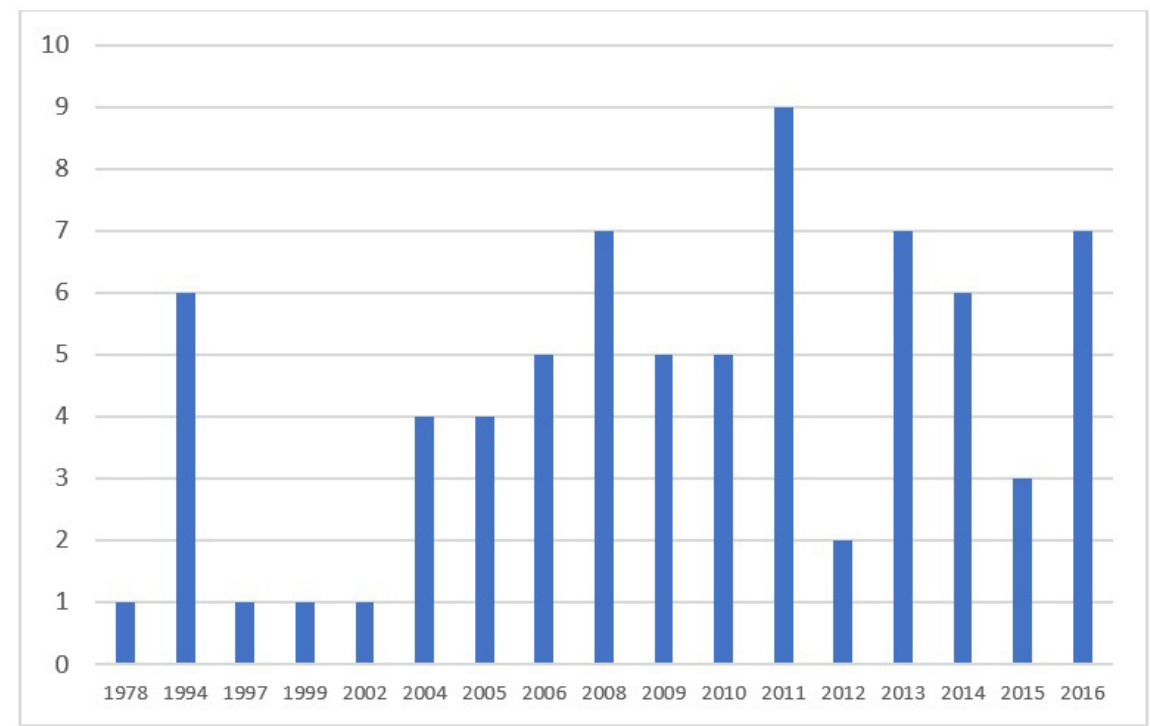

Figura 3. Frecuencias de años de publicación

Como se evidencia en la Figura 4, la universidad líder en publicaciones en Colombia de acuerdo con la frecuencia de publicación del autor de correspondencia es la Fundación Universitaria Konrad Lorenz (23), seguida de la Universidad de los Andes, Universidad del Norte y Universidad Icesi (9 cada una), la Universidad del Rosario (5), la Universidad Javeriana, Universidad de Ibagué y Universidad Simón Bolívar (2 cada una), y otras siete universidades cuentan con un artículo publicado.

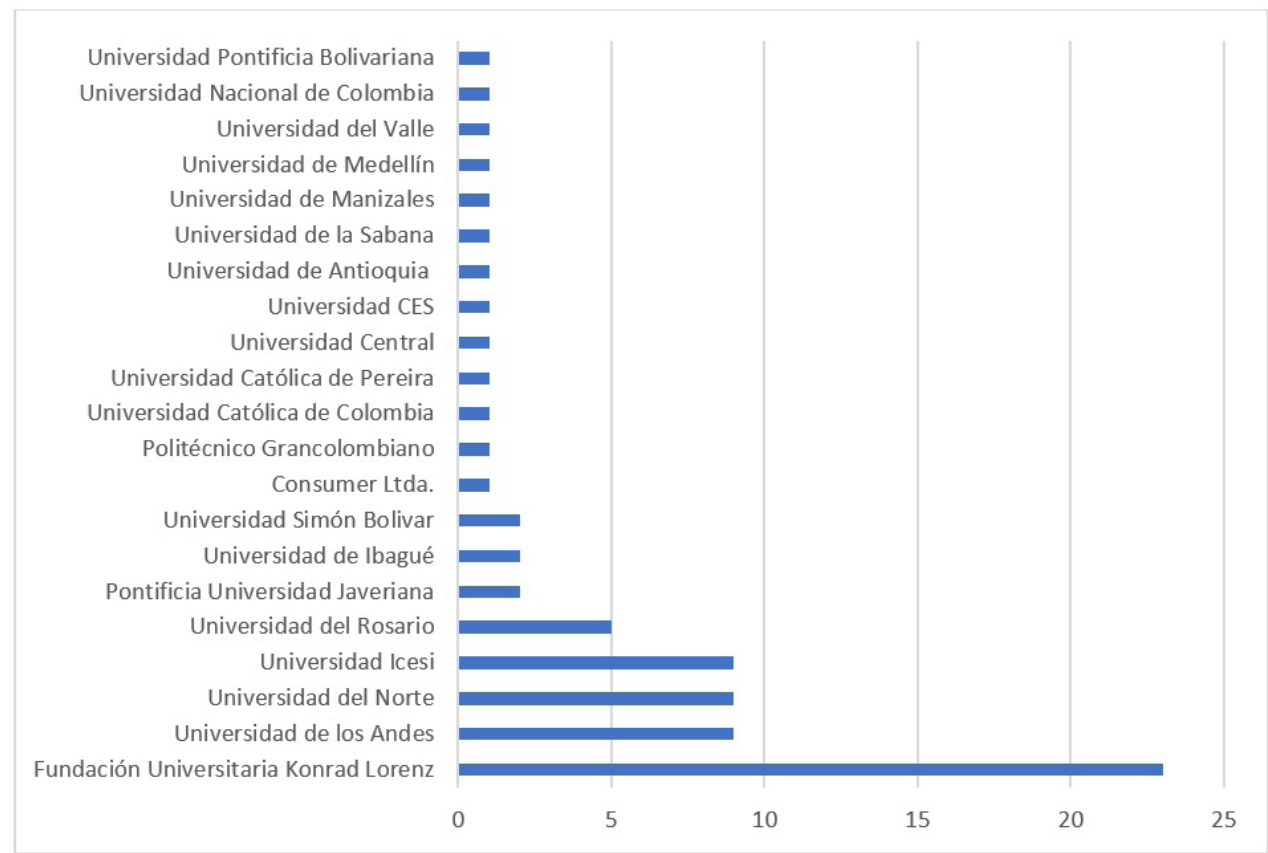

Figura 4. Frecuencias de publicación de artículos por institución 
En la Figura 5 se presenta la producción científica sobre psicología del consumidor por ciudades de Colombia, siendo Bogotá la que mayor producción presenta (63\%), seguida de Cali y Barranquilla (14\% cada una), Medellín (4\%), Ibagué (3\%) y, Pereira y Manizales con un $1 \%$.

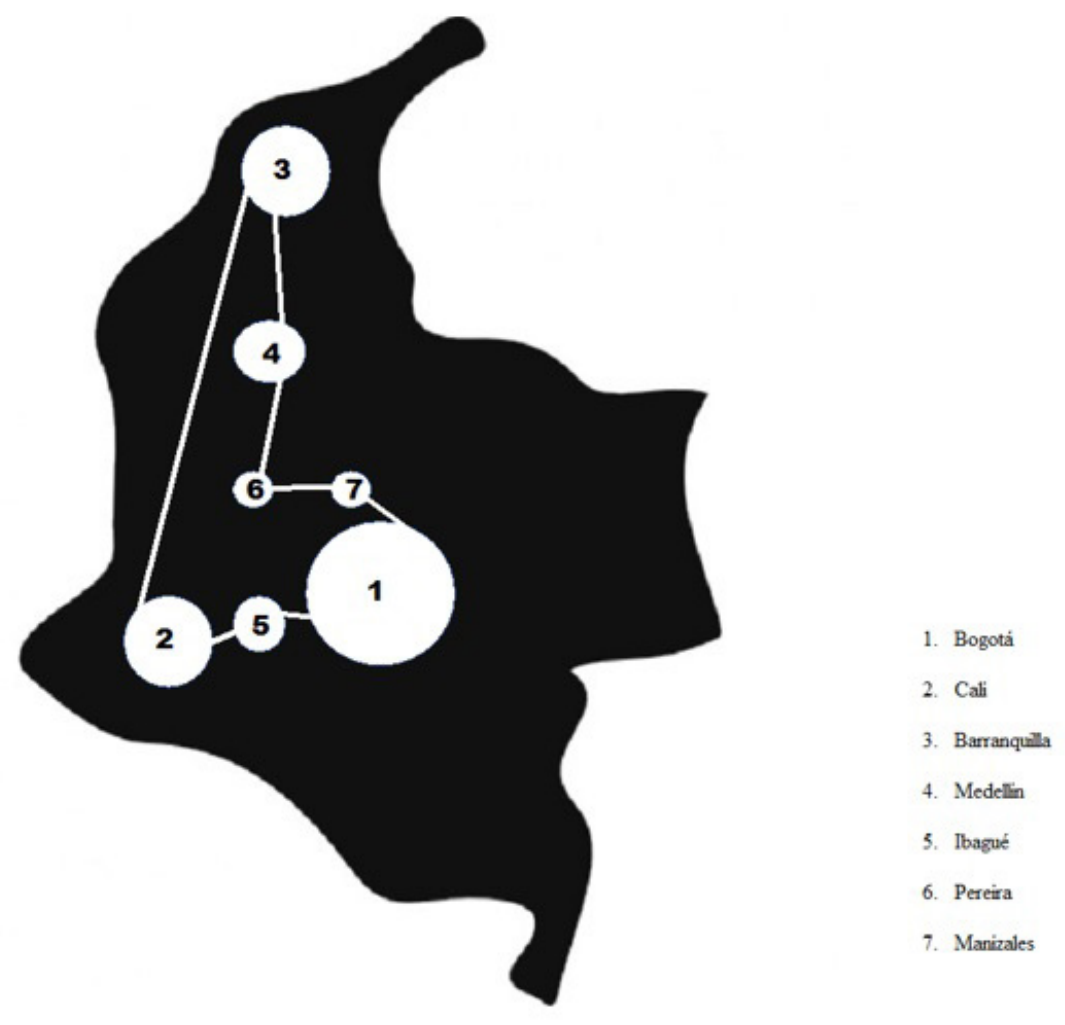

Figura 5. Frecuencias de publicación por ciudades según autor de correspondencia

Respecto a las revistas que más publican artículos en psicología del consumidor en Colombia (ver Figura 6) se observa que Suma Psicológica presenta la frecuencia más alta con 18 artículos; Psicología desde el Caribe y la Revista Latinoamericana de Psicología, 7 cada una; seguidas de Diversitas Perspectivas en Psicología, Pensamiento y Gestión y Universitas Psychologica, con 6 artículos cada una; Estudios Gerenciales con un total de 5; Acta Colombiana de Psicología, Psicogente y la Revista Colombiana de Psicología, con dos artículos cada una. Y cada una de las trece revistas restantes que conforman la muestra con un artículo publicado.

Los tipos de artículos más publicados son los de investigación con $70 \%$, seguido de los correspondientes a reflexiones teóricas con 17\% y, finalmente, los artículos menos publicados son de revisión de literatura con una representación de 12\%.

Respecto al tipo de colaboración de los autores, se encuentra que la colaboración local es la más frecuente con $55 \%$, seguido de artículos de única autoría con $28 \%$, y colaboración nacional con 16\%. Cabe resaltar que la colaboración internacional no se presentó en ninguno de los artículos que conforman la muestra.

Como se observa en la Figura 7, respecto a las palabras claves asociadas al término de psicología del consumidor utilizadas con más frecuencia se encuentran: 
"comportamiento del consumidor" y "psicología del consumidor" con una frecuencia de 11 y 10, respectivamente; en segundo lugar, "publicidad" con una frecuencia de 8, seguida de "preferencias" con 7. "Consumidor" con 5, mientras que "condicionamiento clásico", "consumo", "economía conductual", "mercadeo" y "psicología" obtuvieron una frecuencia de 4.

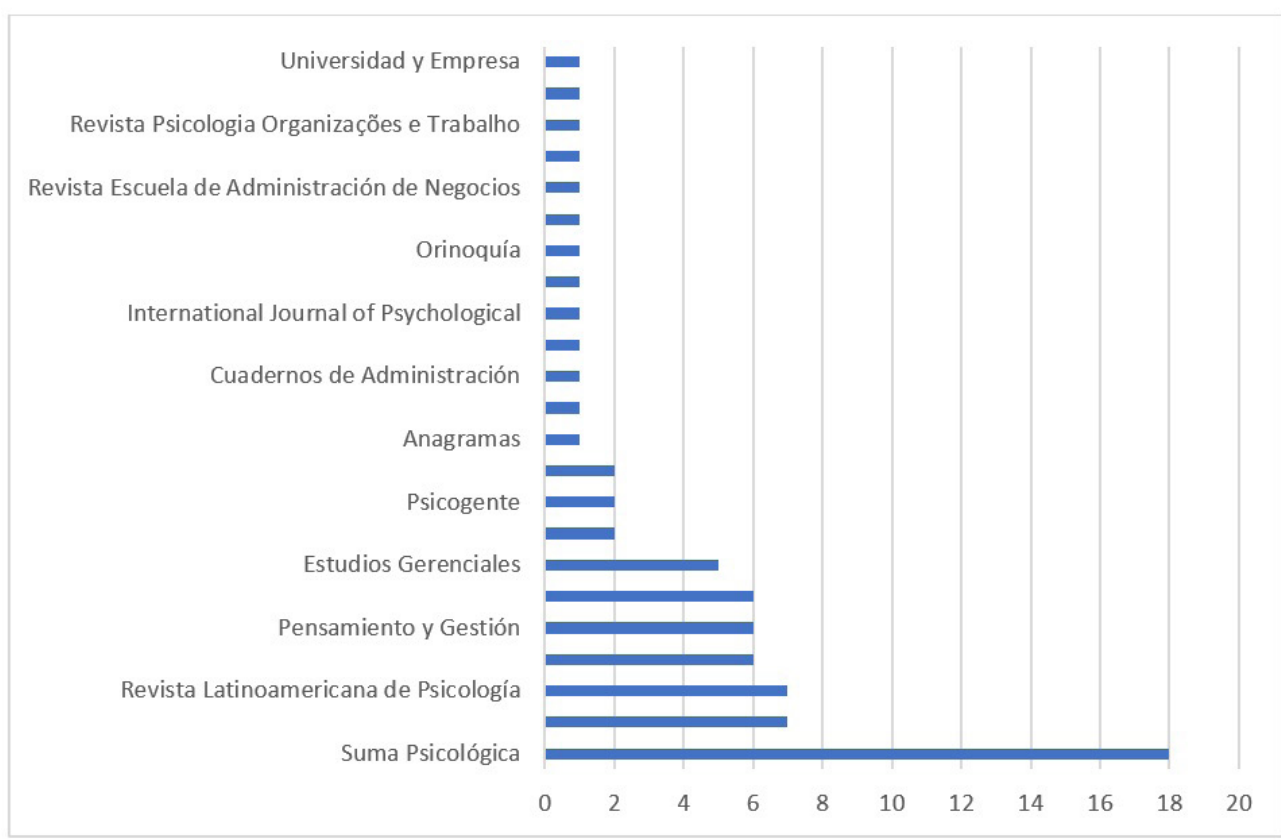

Figura 6. Frecuencias de publicación de artículos según las revistas

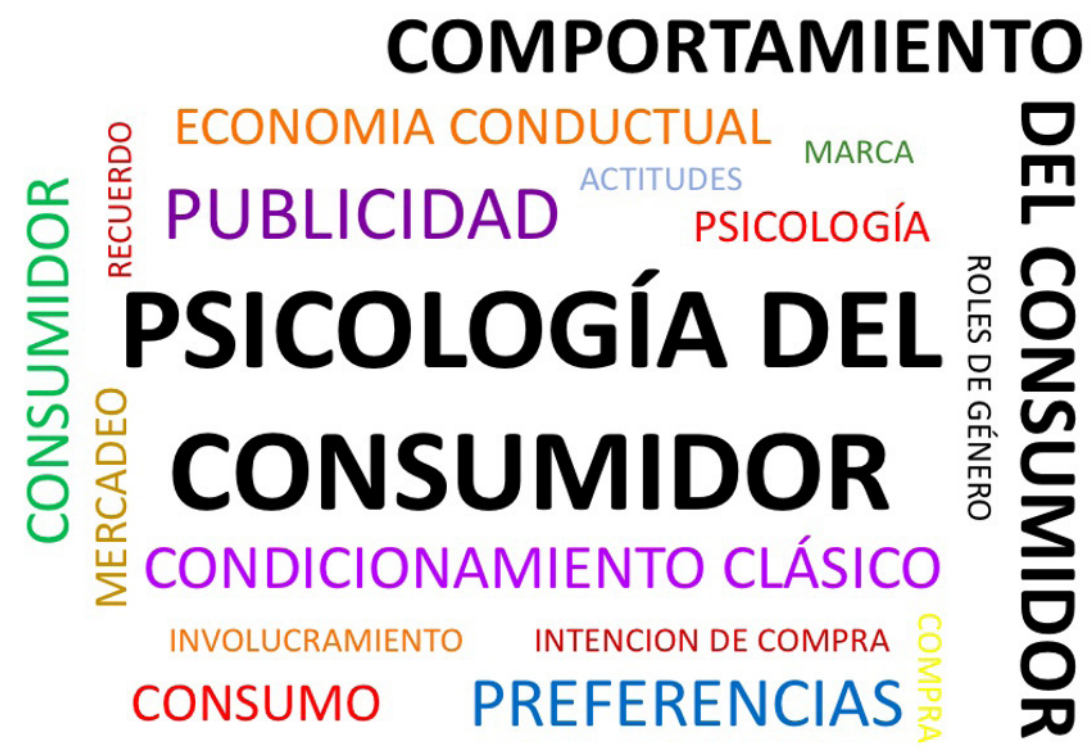

Figura 7. Mapa de palabras clave de los artículos publicados.

Se tuvo en cuenta un segundo grupo de palabras clave que se presentaron con una frecuencia de tres: "actitudes", "compra", "efecto país origen", "intención de compra", "involucramiento", "marca", "recuerdo" y "roles de género". 


\section{Variables de visibilidad e impacto}

La visibilidad se analizó de acuerdo con el número de fuentes presentes en cada base de datos; cabe mencionar que se tuvieron en cuenta todas las fuentes que componen la muestra total sin discriminar el número de citaciones en las bases de datos. En la base de datos de Google Scholar resultaron visibles todas las fuentes que conforman la muestra (100\%), es decir, los 74 artículos; Scopus obtuvo una visibilidad de 20 fuentes, es decir, 20 de los 74 artículos de la muestra total (27,02\%), y Web of Science fue la base con menos fuentes visibles con 8 artículos (10,81\%).

Respecto al impacto, calculado por el número de citas de los artículos que conforman la muestra en cada una de las bases de datos mencionadas, hasta 2017, la base de datos con mayor número de citas fue Google Scholar con una frecuencia de 583 citaciones, seguido de Scopus con 27 citaciones y, finalmente, Web of Science con un total de 9 citaciones. Relacionando la visibilidad de las bases de datos con el impacto de los artículos, se encuentra que por cada fuente con citas incluida en Web of Science hay 2,75 fuentes con citas incluidas en Scopus y 16,25 incluidas con citas en Google Scholar.

Adicionalmente, se analizó de manera detallada, para cada base de datos, los artículos más citados y la frecuencia de citación de cada uno (ver Tabla 1). Para el top de citaciones (lista de artículos más citados) de Google Scholar se tuvieron en cuenta los artículos citados con una frecuencia mayor a 10, y se encontró que un total de 26 artículos fueron citados 10 veces o más. Para el top de citaciones en Scopus se tuvieron en cuenta todas las fuentes que fueron citadas al menos una vez en la base de datos y se encontró que 11 artículos fueron citados al menos una vez en la base de datos. Finalmente, para el top de citaciones de la base de datos Web of Science, al igual que con Scopus, se tomaron todos los artículos citados al menos una vez en la base de datos, y se encontró que 4 artículos fueron citados, con diferentes frecuencias cada uno.

Tabla 1. Top de citaciones en Google Scholar, Scopus y Web of Science

\begin{tabular}{clc}
\hline Número & \multicolumn{1}{c}{ Títulos por base de datos } & Citaciones \\
\hline \multicolumn{1}{c}{ Calidad en el servicio: el cliente incógnito } & 41 \\
\hline 2 & El concepto de consumo socialmente responsable y su medición. Una revisión de la literatura & 32 \\
\hline 3 & Neuroimágenes en la investigación de mercados & 27 \\
\hline 4 & Percepciones del color y de la forma de los empaques: una experiencia de aprendizaje & 26 \\
\hline 5 & Psicología y asuntos económicos: una aproximación al estado del arte & 22 \\
\hline 6 & Representaciones sociales asociadas al consumo hedónico de alimentos en restaurantes & 22 \\
\hline 7 & La psicología del consumidor: una discusión de su estado actual y aportes al mercadeo & 21 \\
\hline 8 & Características psicológicas de consumidores de cibersexo: una aproximación & 16 \\
\hline 9 & $\begin{array}{l}\text { ¿Usted mismo, S.A.? O el posicionamiento profesional en el mercado de trabajo. Reflexiones } \\
\text { desde el marketing y el comportamiento del consumidor }\end{array}$ & 16 \\
\hline 10 & Aproximación teórica al modelo psico económico del consumidor & 14 \\
\hline 11 & $\begin{array}{l}\text { Compra impulsiva en el homosexual masculino. Estudio exploratorio en la ciudad de } \\
\text { Barranquilla (Colombia) }\end{array}$ & 14 \\
\hline 12 & Dimensión integral e interdisciplinaria del concepto de comportamiento del consumidor & 13 \\
\hline
\end{tabular}




\begin{tabular}{|c|c|c|}
\hline Número & Títulos por base de datos & Citaciones \\
\hline \multicolumn{3}{|c|}{ Google Scholar (punto de corte: 10 citas) } \\
\hline 13 & $\begin{array}{l}\text { Factores compensatorios y no compensatorios que influyen en la decisión de compra de } \\
\text { productos culinarios en la categoría de caldos concentrados, en consumidores de la ciudad } \\
\text { de Barranquilla }\end{array}$ & 13 \\
\hline 14 & $\begin{array}{l}\text { Involucramiento de producto y lealtad de marca para productos de consumo masivo en } \\
\text { Bogotá D. C. }\end{array}$ & 13 \\
\hline 15 & Prácticas de consumo y estilo de vida de la población LGTB de Bogotá & 13 \\
\hline 16 & $\begin{array}{l}\text { Técnicas experimentales aplicadas al condicionamiento clásico de preferencias en el } \\
\text { comportamiento del consumidor }\end{array}$ & 13 \\
\hline 17 & $\begin{array}{l}\text { Consumo ideológico: creencias sobre la política de seguridad democrática e imagen del } \\
\text { presidente Álvaro Uribe Vélez }\end{array}$ & 12 \\
\hline 18 & Prueba experimental del efecto de preferencia de marcas pioneras & 12 \\
\hline 19 & Análisis psicológico del posicionamiento publicitario una propuesta cuantitativa & 11 \\
\hline 20 & El comportamiento del consumidor de cine: una revisión preliminar de la literatura & 11 \\
\hline 21 & Estereotipos y roles de género utilizados en la publicidad transmitida a través de la televisión & 11 \\
\hline 22 & La psicología del consumidor & 11 \\
\hline 23 & Nivel de sexismo en consumidores y su valoración sexista de la publicidad & 11 \\
\hline 24 & $\begin{array}{l}\text { Validación del modelo psico económico del consumidor. Análisis causal con ecuaciones } \\
\text { estructurales }\end{array}$ & 11 \\
\hline 25 & $\begin{array}{l}\text { Estrategias metodológicas para el análisis de datos textuales: aplicaciones en psicología del } \\
\text { consumidor }\end{array}$ & 10 \\
\hline 26 & $\begin{array}{l}\text { La compra impulsiva y el materialismo en los jóvenes: estudio exploratorio en estudiantes } \\
\text { universitarios de Barranquilla (Colombia) }\end{array}$ & 10 \\
\hline \multicolumn{3}{|c|}{ Scopus } \\
\hline 1 & Representaciones sociales asociadas al consumo hedónico de alimentos en restaurantes & 6 \\
\hline 2 & $\begin{array}{l}\text { Consumo ideológico: creencias sobre la política de seguridad democrática e imagen del } \\
\text { presidente Álvaro Uribe Vélez }\end{array}$ & 4 \\
\hline 3 & Nivel de sexismo en consumidores y su valoración sexista de la publicidad & 4 \\
\hline 4 & $\begin{array}{l}\text { Estrategias metodológicas para el análisis de datos textuales: aplicaciones en psicología del } \\
\text { consumidor }\end{array}$ & 3 \\
\hline 5 & $\begin{array}{l}\text { Percepción de riesgo y compras por internet: su relación con la personalidad y el tipo de } \\
\text { producto }\end{array}$ & 3 \\
\hline 6 & $\begin{array}{l}\text { Patrones de elección de marca en función de los cambios en los niveles de refuerzo utilitario } \\
\text { e informacional en categorías de productos de consumo masivo }\end{array}$ & 2 \\
\hline 7 & $\begin{array}{l}\text { Diseño y análisis psicométrico de un instrumento que evalúa competencias básicas en } \\
\text { Economía y Finanzas: una contribución a la educación para el consumo }\end{array}$ & 1 \\
\hline 8 & Estereotipos y roles de género utilizados en la publicidad transmitida a través de la televisión & 1 \\
\hline 9 & Función de las consecuencias en la alteración de jerarquías de marcas experimentales & 1 \\
\hline 10 & Influencia del advergaming y el advertising en la recordación y reconocimiento de una marca & 1 \\
\hline 11 & $\begin{array}{l}\text { Prácticas alimentarias y significados de los alimentos light de familias de un sector urbano } \\
\text { de Bogotá }\end{array}$ & 1 \\
\hline \multicolumn{3}{|c|}{ Web of Science } \\
\hline 1 & $\begin{array}{l}\text { Consumo ideológico: creencias sobre la política de seguridad democrática e imagen del } \\
\text { presidente Álvaro Uribe Vélez }\end{array}$ & 4 \\
\hline 2 & Behavioral Perspective Model: Conceptual analysis and review & 2 \\
\hline 3 & Estereotipos y roles de género utilizados en la publicidad transmitida a través de la televisión & 2 \\
\hline 4 & Influencia del advergaming y el advertising en la recordación y reconocimiento de una marca & 1 \\
\hline
\end{tabular}




\section{Variables de Colciencias}

Finalmente, complementando la información bibliométrica, se realizó una revisión adicional de los ocho autores de correspondencia más importantes en la base de datos de grupos de investigación de Colciencias (GRUPLAC) con el fin de conocer los grupos de investigación a los que están asociados, la clasificación de los grupos y la categoría individual como investigador de cada autor (ver Tabla 2). En términos generales, se aprecia que hay una relación directamente proporcional entre la productividad (frecuencia de autores de correspondencia), la categoría del grupo en el GRUPLAC de Colciencias a los que están adscritos como investigadores y la categoría individual de investigador de cada uno de los autores. Por ejemplo, Marithza Sandoval Escobar es líder del grupo de investigación de Psicología del Consumidor, clasificado en A1 (la más alta) y su categoría individual es investigadora senior. Idaly Barreto también se encuentra asociada al grupo de Psicología del Consumidor (A1), y en la categoría individual investigadora senior. En tercer lugar, se encuentra Andrés M. Pérez-Acosta, quien hace parte del grupo de investigación de Estudios en Ciencias del Comportamiento (A1), y su categoría individual es investigador asociado.

Tabla 2. Revisión de clasificaciones de Colciencias para grupos y autores

\begin{tabular}{llcc}
\hline \multicolumn{1}{c}{ Autor } & \multicolumn{1}{c}{ Grupo de Investigación } & $\begin{array}{c}\text { Clasificación } \\
\text { del grupo }\end{array}$ & Categoría individual \\
\hline Marithza Sandoval Escobar & Psicología del consumidor & A1 & Investigador Sénior \\
\hline Idaly Barreto & Psicología del consumidor & A1 & Investigador Sénior \\
\hline Andrés M. Pérez-Acosta & $\begin{array}{l}\text { E. C. Estudios en Ciencias del } \\
\text { Comportamiento }\end{array}$ & A1 & Investigador Asociado \\
\hline Ana María Arboleda Arango & N/A & N/A & Investigador Sénior \\
\hline María Mercedes Botero & $\begin{array}{l}\text { Investigación en Psicología del } \\
\text { Consumidor }\end{array}$ & B & Investigador Júnior \\
\hline Andrea Velandia-Morales & Psicología del Consumidor & A1 & Investigador Asociado \\
\hline Julio Eduardo Cruz & E. C. Estudios en Ciencias del & A1 & N/A \\
\hline Jim Walters & Comportamiento & N/A & N/A \\
\hline
\end{tabular}

\section{Discusión}

El presente estudio logra complementar y profundizar los hallazgos del análisis bibliométrico de artículos sobre psicología del consumidor publicados en castellano, realizado por Chilito et al. (2010), con resultados específicos de la producción científica en psicología del consumidor publicada por investigadores afiliados a instituciones colombianas. Además, en la presente investigación se efectuó adicionalmente un análisis del impacto de los artículos medido por citaciones, por el cual se aprecia que la producción nacional ha crecido sostenidamente en las últimas décadas y que ya comienza a tener impacto y reconocimiento en el escenario científico.

Los resultados permitieron identificar algunos aportes importantes dados por las instituciones e investigadores en Colombia. En primera instancia, señalan que la institución universitaria que más publicaciones ha realizado en el campo de la psicología del consumidor en Colombia es la Fundación Universitaria Konrad Lorenz; situación que en gran medida obedece a que dicha institución fue pionera en Colombia en ofrecer postgrados en Psicología del Consumidor y, por tanto, en participar de manera decisiva en la reflexión e investigación de esta área de la psicología. Adicionalmente, 
Los autores de correspondencia que han publicado con mayor frecuencia forman parte de grupos de investigación clasificados en COLCIENCIAS y son investigadores de gran producción y trayectoria en el ámbito nacional. En ese sentido, se valida la política que llevó a priorizar la medición y fortalecimiento de los grupos de investigación en Colombia, con miras a generar ambientes de cooperación y mayor impacto de los investigadores individuales (ver Colciencias, Gobierno de Colombia, 2018). en esta Fundación se edita la Revista científica Suma Psicológica, publicación en la que los autores de los artículos que conforman la muestra han realizado más publicaciones hasta la fecha.

Los autores de correspondencia que han publicado con mayor frecuencia forman parte de grupos de investigación clasificados en COLCIENCIAS y son investigadores de gran producción y trayectoria en el ámbito nacional. En ese sentido, se valida la política que llevó a priorizar la medición y fortalecimiento de los grupos de investigación en Colombia, con miras a generar ambientes de cooperación y mayor impacto de los investigadores individuales (ver Colciencias, Gobierno de Colombia, 2018).

Con respecto al tipo de colaboración entre los autores de los artículos que componen la muestra, fue evidente que el más frecuente fue el de colaboración institucional, es decir, las co-autorías de la misma institución académica; además, se observa que los artículos fueron publicados principalmente en revistas locales, lo que sugiere la necesidad de promover investigaciones con colaboración internacional y publicar en revistas indexadas de carácter internacional. para dar mayor visibilidad e impacto a la investigación colombiana en psicología del consumidor.

Es importante mencionar la relevancia de la base de datos Google Scholar para la detección de los artículos, ya que todas las fuentes seleccionadas fueron visibles allí; además, tal visibilidad ofrece potencial medida de impacto para todas las fuentes.

En lo que se refiere a las palabras claves, "Psicología del Consumidor" es la expresión más frecuente, lo que verifica que la muestra está bien diferenciada con respecto a su contexto más general, es decir, el campo interdisciplinario del comportamiento del consumidor (ver Jansson-Boyd, 2010). Teniendo en cuenta que "Psicología" y "Publicidad" son de las palabras más relevantes en el listado, se puede reafirmar que las disciplinas a las que hacen alusión son muy pertinentes para la investigación del comportamiento del consumidor (ver Schiffman \& Lazar, 2010). Por último, con base en la frecuencia de la palabra clave "condicionamiento clásico", se puede inferir que el enfoque conductual (ver Parrado, 2013) es una de las perspectivas de la psicología más involucradas en la investigación relativa al consumidor en Colombia.

\section{Agradecimiento}

Los autores de este trabajo queremos agradecer al profesor Jim Walters (Fundación Universitaria Konrad Lorenz), por sus valiosas orientaciones académicas. 


\section{Referencias}

American Psychological Association. (2019). Divisions of APA: Society for Consumer Psychology. Recuperado de: http://www.apa.org/about/division/div23.aspx

Chilito Pinto, M. A., Rodríguez Rojas, P. A., Plata-Caviedes, T., \& Pérez-Acosta, A. M. (2010). Artículos académicos de psicología del consumidor publicados en castellano: Una revisión bibliométrica. Revista Electrónica de Motivación y Emoción, 13(35-36), 1-18. Recuperado de: http://reme.uji.es/articulos/numero35/article11/article11.pdf

Colciencias, Gobierno de Colombia. (2018). Modelo de Medición de Grupos de Investigación, Desarrollo Tecnológico o de Innovación y de Reconocimiento de Investigadores del Sistema Nacional de Ciencia, Tecnología e Innovación, Año 2018. Bogotá: Departamento Administrativo de Ciencia, Tecnología e Innovación, Colciencias. Dirección de Fomento a la Investigación. Recuperado de: https://minciencias.gov.co/sites/ default/files/upload/convocatoria/4. anexo 1. documento conceptual del modelo de reconocimiento y medicion de grupos de investigacion 2018.pdf

Jansson-Boyd, C. (2010). Consumer psychology. New York: McGraw Hill. Recuperado de: $\quad$ http://www.umpalangkaraya.ac.id/dosen/dwisariusop/wp-content/uploads/2016/11/Consumer-Psychology.pdf

Parrado Corredor, F. (2013). J. B. Watson y la publicidad, los inicios de la psicología del consumidor. Revista Colombiana de Psicología, 22(2), 401-406. Recuperado de: http://www.scielo.org.co/pdf/rcps/v22n2/v22n2a13.pdf

Raiteri, M. D. (2016). El comportamiento del consumidor actual (Tesis de investigación). Mendoza, Argentina: Universidad Nacional de Cuyo. Recuperado de: http://bdigital.uncu.edu.ar/objetos digitales/8046/raiteri-melisa-daniela.pdf

Rodríguez Santoyo, A. R. (2012). Antología comportamiento del consumidor. Recuperado de: http://www.eumed.net/libros-gratis/2012b/1217/indice.htm

Sandoval Escobar, M. (1994). La psicología del consumidor: una discusión de su estado actual y aportes al mercadeo. Suma Psicológica, 1(2), 163-176. doi: http:// dx.doi.org/10.14349/sumapsi1994.294

Sandoval-Escobar, M. (2018). Psicología del consumidor: temas escogidos de investigación, Bogotá: Fundación Universitaria Konrad Lorenz.

Schiffman, L.G \& Lazar, L. (2010). Comportamiento del consumidor. México. Pearson. 